\title{
Effects of convection and solid wall on the diffusion in microscale convection flows
}

\author{
Jun Zhang, Jing Fan, ${ }^{a}$ and Fei Fei \\ Laboratory of High Temperature Gas Dynamics, Institute of Mechanics, Chinese Academy of Sciences, \\ Beijing 100190, China
}

(Received 9 June 2010; accepted 29 November 2010; published online 21 December 2010)

\begin{abstract}
The diffusive transport properties in microscale convection flows are studied by using the direct simulation Monte Carlo method. The effective diffusion coefficient $D^{*}$ is computed from the mean square displacements of simulated molecules based on the Einstein diffusion equation $D^{*}=\left\langle\Delta x^{2}(t)\right\rangle / 2 t$. Two typical convection flows, namely, thermal creep convection and RayleighBénard convection, are investigated. The thermal creep convection in our simulation is in the noncontinuum regime, with the characteristic scale of the vortex varying from 1 to 100 molecular mean free paths. The diffusion is shown to be enhanced only when the vortex scale exceeds a certain critical value, while the diffusion is reduced when the vortex scale is less than the critical value. The reason for phenomenon of diffusion reduction in the noncontinuum regime is that the reduction effect due to solid wall is dominant while the enhancement effect due to convection is negligible. A molecule will lose its memory of macroscopic velocity when it collides with the walls, and thus molecules are hard to diffuse away if they are confined between very close walls. The RayleighBénard convection in our simulation is in the continuum regime, with the characteristic length of 1000 molecular mean free paths. Under such condition, the effect of solid wall on diffusion is negligible. The diffusion enhancement due to convection is shown to scale as the square root of the Péclet number in the steady convection regime, which is in agreement with previous theoretical and experimental results. In the oscillation convection regime, the diffusion is more strongly enhanced because the molecules can easily advect from one roll to its neighbor due to an oscillation mechanism. (c) 2010 American Institute of Physics. [doi:10.1063/1.3528310]
\end{abstract}

\section{INTRODUCTION}

The diffusive transport in fluid flows is an important process in many fields such as chemical engineering, biophysics, oceanography, and astrophysics. Knowledge of how the fluid flow affects the diffusion is of practical and fundamental importance. On the one hand, in many technological processes, it is desired to enhance or reduce the transport to optimize the process condition. On the other hand, the study on transport mechanism contributes to a deeper understanding of the fluid mechanics, and it may help us propose more reasonable transport models for complex flows.

In 1953, Taylor ${ }^{1}$ first showed the enhancement of longitudinal diffusion in the Poiseuille flow confined in a cylindrical tube. In the recent two decades, many efforts have been devoted to the understanding of diffusive transport in convection flows. A well-known viewpoint in the continuum regime is that the diffusion will be enhanced due to convection with respect to molecular diffusion. With the development of the microelectromechanical system (MEMS), more and more attention has been paid to microscale flows. ${ }^{2}$ An interesting question is whether the diffusion is enhanced or not in convection flows where there are merely vortices whose length scale is several or dozens of molecular mean free paths. In such noncontinuum regime, the solid wall may

\footnotetext{
a) Author to whom correspondence should be addressed. Electronic mail: jfan@imech.ac.cn.
}

play an important role on preventing diffusion. Therefore, it is necessary to study the effects of solid wall as well as convection on diffusion in microscale convection flows. A convenient model for this study is the thermal creep convection $^{3,4}$ usually formed in the rarefied gas regime. The thermal creep convection is induced by creep mechanism, ${ }^{5}$ which can be explained on the basis of kinetic theory as follows. Consider a gas with a temperature gradient along a solid wall. Because hotter molecules impart more tangential momentum to the wall than colder ones, a shear stress is exerted on the wall, with the gas flowing from the colder to the hotter part as a reaction force. In thermal creep convection, various vortices with their scales varying from several to hundreds of the molecular mean free paths may form in accordance with the temperature distributions along the wall. In this paper, the dependence of diffusion on vortex scale is investigated in thermal creep convection by using direct simulation Monte Carlo (DSMC) method, ${ }^{6}$ which is a direct molecular simulation method based on kinetic theory. The DSMC method was first successfully applied to many highspeed problems in the context of aerospace engineering and was then extended to investigate the mechanism of various fundamental flow phenomena at the molecular level.

Meanwhile, we also study the diffusive transport properties in continuum regime, such as in Rayleigh-Bénard (RB) convection, ${ }^{7}$ where vortex structures will form above the onset of thermal instability. RB convection is a good model system for a comprehensive study on the transport, since 


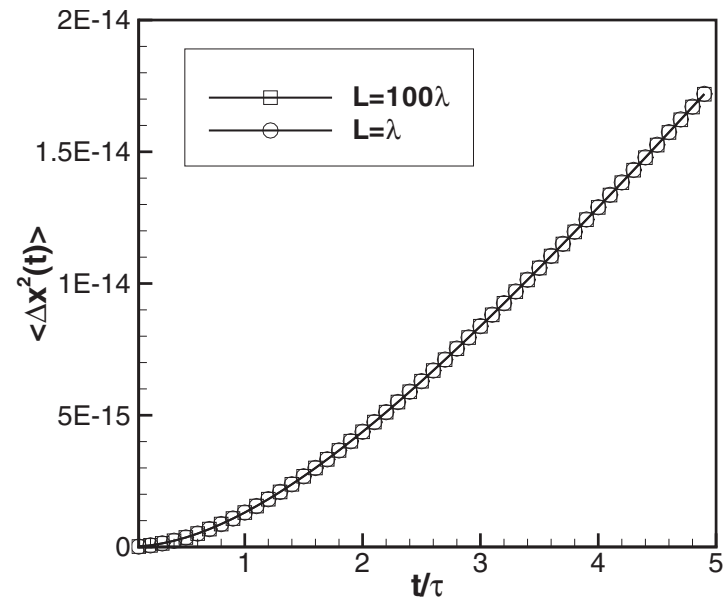

FIG. 1. Molecular mean square displacements vs time in an equilibrium state at $273 \mathrm{~K}$

various types of convective flows from steady to turbulent can be generated as the Rayleigh number increases. As a result, the transport rates vary over a wide range. Recently, the molecular dynamics (MD) and DSMC method have been successfully applied to the simulation of RB instability. ${ }^{8-17}$ Vannitsem and Mareschal ${ }^{18}$ first studied the self-diffusive transport of molecules in a two-dimensional RB convection flow based on the Einstein diffusion equation by employing MD technique. However, their study was restricted to the time-independent regime due to the limitation of computational power at that time. Here we will use the DSMC method to investigate the diffusion in RB convection flows. For gases, DSMC can be applied to the problem with its length scale many orders of magnitude greater than those to which the standard MD techniques are currently applied. Therefore, RB convection for large Rayleigh number is easier to be obtained by DSMC than by MD with the same computation power. In this paper, we study the diffusion enhancement in not only the steady RB convection but also the oscillation convection.

Our objective is twofold; one is diffusive transport properties in convection flows in noncontinuum regime, such as thermal creep convection. By varying the scale of convection vortex, we will show the dependence of diffusive properties on the vortex scale in the noncontinuum regime. Another is the diffusive transport properties in convection flows in the continuum regime, such as $\mathrm{RB}$ convection. We perform a comprehensive study covering both the steady convection and the oscillation convection. Based on these studies, the effects of convection and solid wall on diffusion in different flow regimes will be shown in the Secs. III and IV.

\section{COMPUTATIONAL METHOD AND VERIFICATION IN EQUILIBRIUM STATE}

In the continuum viewpoint, the transport of particles is governed by the convection-diffusion equation, that is,

$$
\partial_{t} c=D_{m} \nabla^{2} c-\mathbf{v} \cdot \nabla c,
$$

where $c(\mathbf{r}, t)$ is the particle density, $D_{m}$ is the molecular diffusion coefficient, and $\mathbf{v}(\mathbf{r}, t)$ is the velocity field. For a long

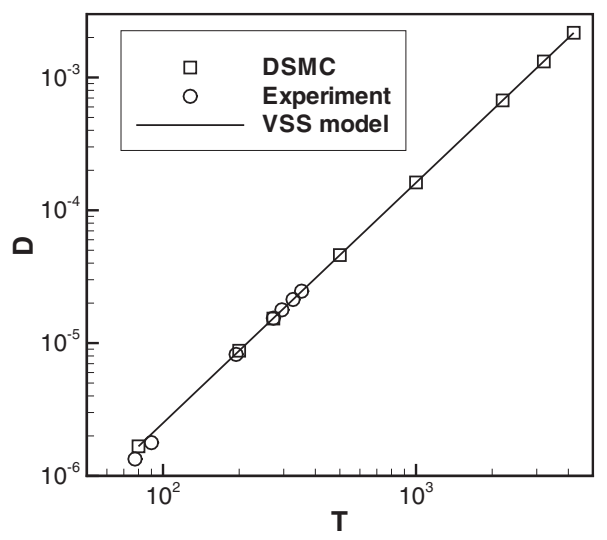

FIG. 2. Diffusion coefficient vs temperature for argon gas.

enough time scale, the particle transport in convection flows could be described by an effective diffusion process, that is,

$$
\partial_{t} c=D^{*} \nabla^{2} c,
$$

where $D^{*}$ is the effective diffusion coefficient. This has been validated by Gollub and Solomon ${ }^{19}$ based on experiment in $\mathrm{RB}$ convection. In this way, the normalized diffusion enhancement due to convection with respect to molecular diffusion can be expressed as

$$
\delta_{1}=\left(D^{*}-D_{m}\right) / D_{m} .
$$

In this paper, we want to study the diffusive transport properties in microscale convection flows. Besides the convection effect, the solid boundary wall and temperature variation in the field may also affect the diffusion. We employ Einstein's diffusion equation ${ }^{20}$ to calculate the effective diffusion coefficient, that is,

$$
\left\langle\Delta x^{2}(t)\right\rangle=2 D t, \quad t \rightarrow \infty,
$$

where $\left\langle\Delta x^{2}(t)\right\rangle$ is the particle's mean square displacements in one-dimensional direction. If $\left\langle\Delta x^{2}(t)\right\rangle$ is proportional to time when the time scale is long enough, then the transport of particles can be considered as an effective diffusion process. Consequently, the molecular diffusion coefficient $D_{m}$ and effective diffusion coefficient $D^{*}$ can be obtained based on Eq. (4) in equilibrium state and convective flows, respectively. In this paper, the diffusion coefficients are calculated by using Eq. (4) and the DSMC method. The fundamental idea of DSMC is to track a large number of statistically representa-

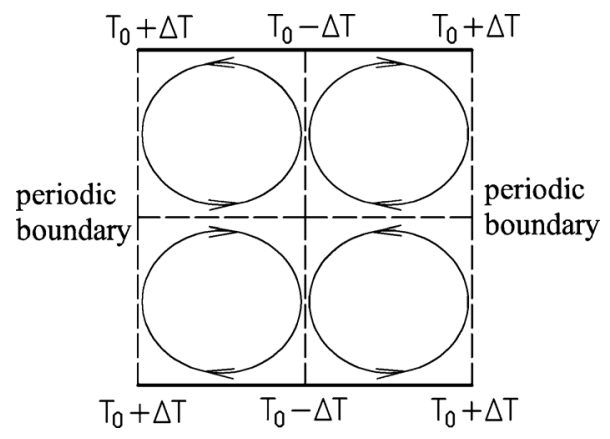

FIG. 3. Thermal creep convection model in our simulation. 


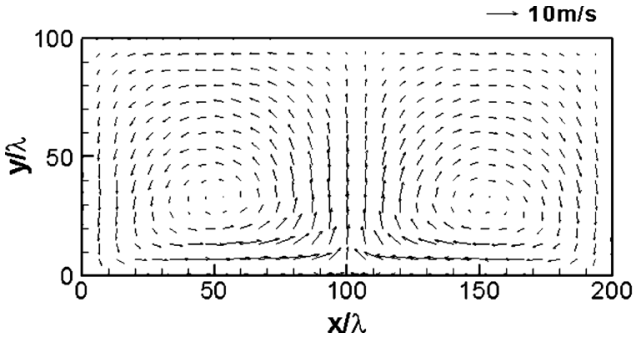

(a) $L=100 \lambda$

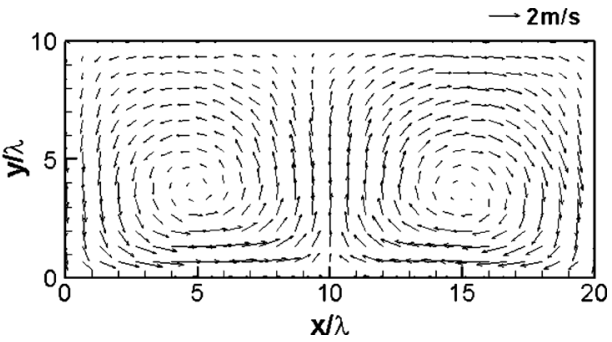

(b) $L=10 \lambda$

FIG. 4. Distributions of velocity vector for in thermal creep convection.

tive molecules, with their motions and intermolecular collisions assumed uncoupled within small time intervals. The molecular collision models are determined from the wellknown Chapman-Enskog theory and phenomenological method.

In order to verify the computational method and program code used in this paper, the diffusion coefficient of argon in equilibrium state is first calculated. The calculated results are compared with the theoretical value and experimental data. The computational domain is a two-dimensional square, and each boundary condition of which is assumed as periodic. The introduction of periodic boundaries is equivalent to considering an infinite, space-filling array of identical copies of the simulation region. In the process of simulation, a molecule that leaves the simulation region through a particular boundary immediately reenters the region through the opposite boundary. It should be noted that the real molecular displacements are needed for the calculation of transport coefficients when the Einstein diffusion equation is used. For convenience, two types of coordinates are recorded for every molecule in our simulation: one is used to determine the location in the simulation domain, while the other is the real coordinate, which is used to calculate the displacement. At the initial time, the two types of coordinates coincide into the same one. Every time one molecular gets through a periodic boundary, the length of the simulation domain is added to or subtracted from its real coordinate.

Figure 1 shows the molecular mean square displacements in the $x$ direction when the gas temperature is $273 \mathrm{~K}$. Here the variable soft sphere (VSS) model ${ }^{6}$ is employed to describe the interaction of molecular collisions. The side lengths in two different simulations are $100 \lambda$ and $\lambda$, respectively, where $\lambda$ is the molecular mean free path. Time is normalized by the molecular mean collision time $\tau$. For $t / \tau<1$, the number of molecular collisions are very small, so the mean square displacement behaves as it would for free particles, $\left\langle\Delta x^{2}(t)\right\rangle=\bar{c}^{2} t^{2}$, where $\bar{c}$ is the average thermal velocity of molecules. For $t / \tau>3$ the mean square displacement varies linearly with time, which means that after enough collisions each molecule can be regarded as a Brownian particle. Consequently, the diffusion coefficients can be obtained from Eq. (4). Both of the diffusion coefficients obtained from these two simulations are 1.54 $\times 10^{-5} \mathrm{~m}^{2} \mathrm{~s}^{-1}$, which compares well with the experimental data and theoretical value for VSS model. These results show that the size of simulation domain does not affect the simu- lation results so long as the number of simulated molecules is large enough to reduce the statistical scatter to an acceptable level.

The relation of diffusion coefficient versus temperature is also validated. As shown in Fig. 2, the diffusion coefficients computed from the DSMC simulations are consistent with the theoretical values for the VSS model in the whole range of temperature. Meanwhile, the experimental data ${ }^{21}$ support the DSMC results in the range of 200-300 K. As we know, there is no available experimental data of diffusion coefficient for argon gas at higher temperature. Fortunately, this situation does not affect our study. Our objective is to study the effect of convection and boundary wall on transport diffusive properties by comparing the effective diffusion coefficients obtained by DSMC simulations with that in a static state at the same temperature with the same molecular collision model. In the following two sections, the diffusive transport properties in thermal creep convection and RB convection are studied. The simulation gas is argon and the VSS model is used to describe the interactions between gaseous molecules.

\section{DIFFUSIVE TRANSPORT PROPERTIES IN THERMAL CREEP CONVECTION}

The thermal creep flow resulting from the presence of temperature gradients along boundary walls was first discovered by Reynolds and later studied by Maxwell. By far, a number of microscale and nanoscale systems has utilized this

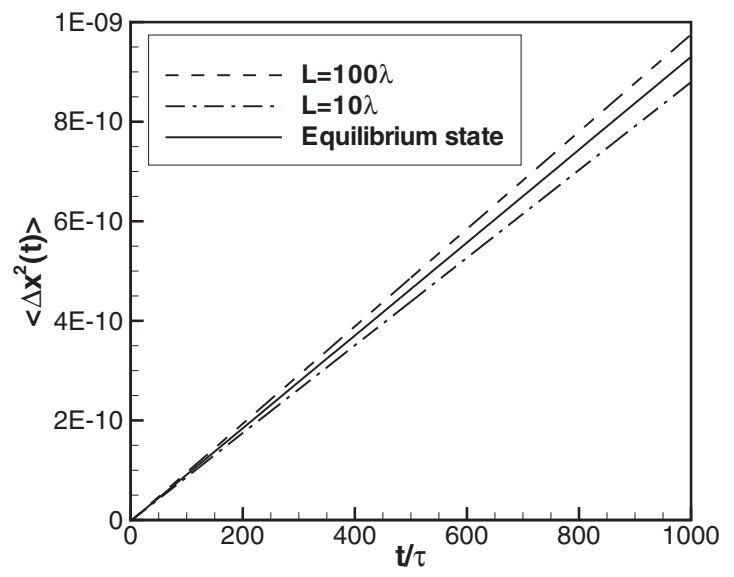

FIG. 5. The mean square displacements vs time in thermal creep convection and equilibrium state. 


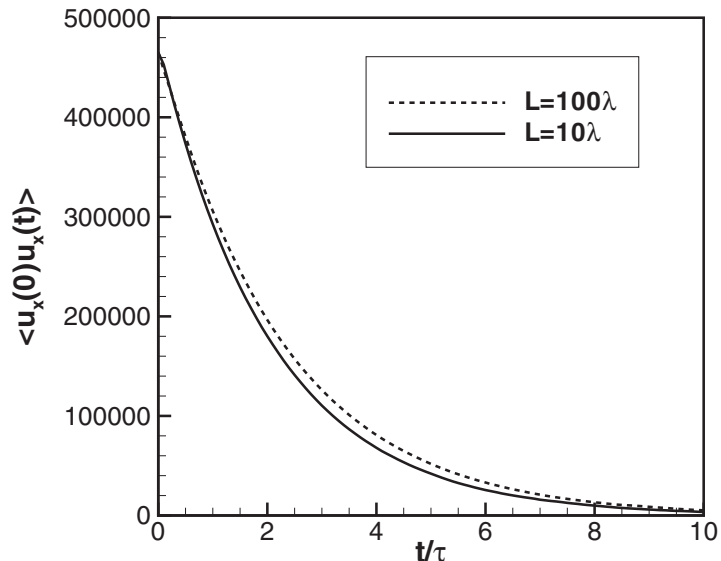

FIG. 6. The molecular velocity autocorrelation function vs time in static state with uniform temperature along the wall.

phenomenon for pumping and propulsion. ${ }^{22}$ In order to know the detailed flow field of thermal creep flows, many different approaches ${ }^{23,24}$ have been proposed to simulate it, and a successful one is DSMC method. Thus, it is tempting to investigate the diffusive transport properties in thermal creep convection by using DSMC method.

We consider a two-dimensional gas flow confined between two horizontal walls (Fig. 3). Two segments of linear temperature distributions are, respectively, imposed at both walls, where diffusive reflections are assumed, while periodic boundary conditions are assumed in the $x$ direction. Consequently, periodic vortex structure along the $x$ direction is formed due to thermal creep mechanism. By adjusting the side length of the simulation domain, we can obtain different scales of convective vortex. Considering the symmetry of the flow structure along the $y$ direction, we only simulate the lower half of the flow region. The two-dimensional computational domain is divided into sampling cells within which the filed quantities are obtained by sampling the molecular information, and each sampling cell contains enough subcells within which collision pairs are selected. The initial state of gas is taken to be uniform with temperature $T_{0}$. Subsequently, the gas is allowed to relax to a steady convective state and sampling is performed thereafter. Here we focus on the molecular mean square displacements and effective diffusion coefficient in the $x$ direction.

First we compare the effective diffusion coefficient of thermal creep convection with different vortex scales, but with the same temperature gradient. According to the kinetic theory, ${ }^{5}$ the velocity caused by thermal creep mechanism is written as

$$
u_{s}=\frac{3}{4} \frac{\mu}{\rho T_{0}}\left(\frac{\partial T}{\partial x}\right)_{y=0},
$$

where $\mu$ is the gas viscosity, while $T_{0}$ and $\rho$ are the average gas temperature and density, respectively. In our simulation, we set $T_{0}=2200 \mathrm{~K}$ and $\rho=0.22 \mathrm{~kg} \mathrm{~m}^{-3}$, and the gas viscosity is constant accordingly. Therefore, the thermal creep velocity is proportional to the temperature gradient along the wall according to Eq. (5). Here we let $(\partial T / \partial x)_{y=0}=40 \mathrm{~K} / \lambda$, that is, the temperature difference is $40 \mathrm{~K}$ for every mean free path.

Figure 4 gives the distributions of velocity vector when the characteristic length of convection vortex equals to $100 \lambda$ and $10 \lambda$, respectively. It clearly shows that a pair of counterrotating vortices is formed due to thermal creep mechanism. Due to the temperature variation in the field, the local molecular diffusion coefficient is different in the field. However, here we study the diffusion ability of system as a whole. It is encouraging that the average temperature in the field is very close to $T_{0}$, so the effect of temperature variation on diffusion can be evaluated by using the molecular diffusion coefficient $D_{m}$ at the average temperature $T_{0}$ in the field. We acknowledge that the diffusion coefficient $D_{m}$ at the average temperature $T_{0}$ may be a little different from the value of average molecular diffusion coefficient in the field, but this effect is negligible in small scale thermal creep convection as the following detail analysis.

Figure 5 shows the evolution of molecular mean square displacement versus time for $L=100 \lambda$ and $L=10 \lambda$. In order to facilitate a comparison, the result in an equilibrium state at temperature $T_{0}$ is also shown. By using Einstein diffusion equation, we can obtain the effective diffusion coefficient

TABLE I. Diffusive transport properties for different convection vortex scales with a temperature gradient of $40 \mathrm{~K} / \lambda$ along the boundary wall in the thermal creep convection. $L$ is the scale of convection vortex. $D_{m}$ is the molecular diffusion calculated for an equilibrium state at temperature $T_{0}, D^{*}$ is the effective diffusion coefficient in thermal creep convection, and $D_{w}$ is the effective diffusion coefficient in the static state with uniform boundary wall. $\delta_{1}$ represents the resultant effect on diffusion, $\delta_{2}$ represents the effect of boundary wall on diffusion, and $\delta_{3}$ represents the effects of convection and temperature variation on diffusion.

\begin{tabular}{|c|c|c|c|c|c|c|}
\hline$L$ & $D_{m} \times 10^{4} \mathrm{~m}^{2} / \mathrm{s}$ & $D^{*} \times 10^{4} \mathrm{~m}^{2} / \mathrm{s}$ & $\begin{array}{c}\delta_{1} \\
(\%)\end{array}$ & $D_{w} \times 10^{4} \mathrm{~m}^{2} / \mathrm{s}$ & $\begin{array}{c}\delta_{2} \\
(\%)\end{array}$ & $\begin{array}{c}\delta_{3} \\
(\%)\end{array}$ \\
\hline $100 \lambda$ & 6.78 & 7.11 & 4.87 & 6.75 & -0.44 & 5.33 \\
\hline $80 \lambda$ & & 6.97 & 2.80 & 6.74 & -0.59 & 3.41 \\
\hline $60 \lambda$ & & 6.84 & 0.89 & 6.72 & -0.88 & 1.78 \\
\hline $50 \lambda$ & & 6.73 & -0.74 & 6.68 & -1.47 & 0.75 \\
\hline $30 \lambda$ & & 6.64 & -2.06 & 6.62 & -2.36 & 0.30 \\
\hline $10 \lambda$ & & 6.41 & -5.46 & 6.40 & -5.60 & 0.16 \\
\hline $5 \lambda$ & & 6.10 & -10.0 & 6.09 & -10.2 & 0.16 \\
\hline$\lambda$ & & 4.14 & -38.9 & 4.13 & -39.1 & 0.24 \\
\hline
\end{tabular}


TABLE II. Diffusive transport properties for $L=100 \lambda$ with different temperature gradients along the boundary wall. The symbols are the same as described in Table I.

\begin{tabular}{cccc}
\hline \hline $\begin{array}{c}\nabla T \\
(\mathrm{~K} / \lambda)\end{array}$ & $D_{w} \times 10^{4} \mathrm{~m}^{2} / \mathrm{s}$ & $D^{*} \times 10^{4} \mathrm{~m}^{2} / \mathrm{s}$ & $\begin{array}{c}\delta_{3} \\
(\%)\end{array}$ \\
\hline 10 & 6.75 & 6.78 & 0.44 \\
20 & & 6.84 & 1.33 \\
30 & & 6.95 & 2.96 \\
40 & & 7.11 & 5.33 \\
\hline \hline
\end{tabular}

$D^{*}$ for $L=100 \lambda$ and $L=10 \lambda$ as $7.11 \times 10^{-4} \mathrm{~m}^{2} / \mathrm{s}$ and $6.41 \times 10^{-4} \mathrm{~m}^{2} / \mathrm{s}$, respectively, while the molecular diffusion coefficient $D_{m}$ at temperature $T_{0}$ is $6.78 \times 10^{-4} \mathrm{~m}^{2} / \mathrm{s}$. Therefore, the normalized variation of effective diffusion with respect to molecular diffusion can be obtained by using Eq. (3). Our simulation gives $\delta_{1}=4.87 \%$ and $\delta_{1}=-5.46 \%$ for $L=100 \lambda$ and $L=10 \lambda$, respectively. It is very interesting to note that the effective diffusion is reduced for $L=10 \lambda$, which seems questionable at first sight because the convection generally enhances the diffusion. However, it should be noted that the solid boundary wall and temperature variation in the field may also affect the diffusion. After careful analysis, we found that the diffusion reduction in small scale convection flows is caused by the dominant effect of boundary wall. We will use the Green-Kubo formula ${ }^{20}$ to elucidate this phenomenon as follows.

The Green-Kubo formula for diffusion coefficient is based on the integrated velocity autocorrelation function, namely,

$$
D=\int_{0}^{\infty}\left\langle u_{x}(0) u_{x}(t)\right\rangle d t .
$$

It is shown that the Green-Kubo formula is completely equivalent to the Einstein relation. In order to clearly show the boundary effect and exclude the convection effect, we simulate two other cases for $L=100 \lambda$ and $L=10 \lambda$ under the same condition as the aforementioned two cases except that there is no temperature gradient along the boundary wall. In these two cases, the flow fields are in static state with uniform wall, and the velocity autocorrelation functions of molecules are shown in Fig. 6. It is obvious that the velocity autocorrelation function for $L=10 \lambda$ is attenuated quickly than that for $L=100 \lambda$. In our simulation, diffusive reflections are assumed at the boundary wall, that is, molecules colliding with the wall rebound with a half-range Maxwellian distribution at the temperature of the corresponding wall. It means that the molecule will lose its velocity memory when it encounters the wall. For $L=10 \lambda$, most of molecules will collide with the wall in several molecular mean collision times, so the velocity correlation function attenuate more quickly than that for $L=100 \lambda$. For convenience, the effective diffusion coefficient in a static state with uniform boundary wall is denoted as $D_{w}$, the normalized variation of $D_{w}$ with respect to $D_{m}$ is denoted as $\delta_{2}$, and the normalized variation of $D^{*}$ with respect to $D_{w}$ is denoted as $\delta_{3}$, namely,
TABLE III. Diffusive transport properties for $L=10 \lambda$ with different temperature gradients along the boundary wall. The symbols are the same as described in Table I.

\begin{tabular}{cccc}
\hline \hline $\begin{array}{c}\nabla T \\
(\mathrm{~K} / \lambda)\end{array}$ & $D_{w} \times 10^{4} \mathrm{~m}^{2} / \mathrm{s}$ & $D^{*} \times 10^{4} \mathrm{~m}^{2} / \mathrm{s}$ & $\begin{array}{c}\delta_{3} \\
(\%)\end{array}$ \\
\hline 40 & 6.40 & 6.41 & 0.16 \\
100 & & 6.42 & 0.31 \\
200 & & 6.42 & 0.31 \\
300 & & 6.43 & 0.47 \\
\hline \hline
\end{tabular}

$$
\begin{aligned}
& \delta_{2}=\left(D_{w}-D_{m}\right) / D_{m}, \\
& \delta_{3}=\left(D^{*}-D_{w}\right) / D_{w} .
\end{aligned}
$$

In this way, $\delta_{2}$ represents the effect of boundary wall on diffusion, and $\delta_{3}$ contains the effects of convection and temperature on diffusion. For $L=10 \lambda$, our simulation result gives $D_{w}=6.42 \times 10^{-4} \mathrm{~m}^{2} / \mathrm{s}, \delta_{2}=-5.60 \%$, and $\delta_{3}=0.16 \%$. It shows that $\left|\delta_{2}\right| \gg\left|\delta_{3}\right|$, so the effect of boundary wall is dominant in such a small scale convection flow, and the effect of convection and temperature variation on diffusion are negligible. For $L=100 \lambda$, our simulation result gives $D_{w}=6.75$ $\times 10^{-4} \mathrm{~m}^{2} / \mathrm{s}, \delta_{2}=-0.44 \%$, and $\delta_{3}=5.33 \%$. It shows that $\left|\delta_{3}\right| \gg\left|\delta_{2}\right|$, so the effect of boundary wall becomes weak, and the effects of convection and temperature variation on diffusion are important. In such moderate scale, it is difficult to separate the convection effect on diffusion from the coupled effects of convection and temperature variation. Further studies can be done in this aspect, but not included in this paper.

From the distinct characteristic of effective diffusion for $L=100 \lambda$ and $L=10 \lambda$, we guess that there is a critical scale which decides whether the effective diffusion is enhanced or reduced. Table I lists our results for different convection vortex scales. It clearly shows that $\delta_{1}$ decreases with decreasing vortex scale, more importantly, there is obviously a certain critical scale $L_{c}$ located between $50 \lambda$ and $60 \lambda$. When $L>L_{c}, \delta_{1}>0$; when $L<L_{c}, \delta_{1}<0$. The variation of effective diffusion with respect to molecular diffusion in thermal creep convection is due to three effects: boundary wall, convection, and temperature variation in the field. When $L<L_{c}$, $\left|\delta_{2}\right|>\left|\delta_{3}\right|$, it means that the diffusion reduction due to the effect of boundary wall is dominant, and thus the resultant effective diffusion is reduced.

It should be noted that the convection velocity in thermal creep convection will be different if the temperature gradient is different. Tables II and III give our results under different temperature gradients for $L=100 \lambda$ and $L=10 \lambda$, respectively. For $L=100 \lambda, \delta_{3}$ increases with increasing temperature gradient, which means that the convection effect plays more and more important role as the convection velocity increases. For $L=10 \lambda, \delta_{3}$ is almost invariable but the boundary effect is dominant. Another point worth notice is that in our simulation, complete diffused reflection is assumed at the boundary wall. If Maxwellian type boundary condition is assumed, that is, part of incident molecules reflects diffusely and the other molecules reflects specularly, the effect of boundary wall on diffusion will become weaker than our results. Based on the 


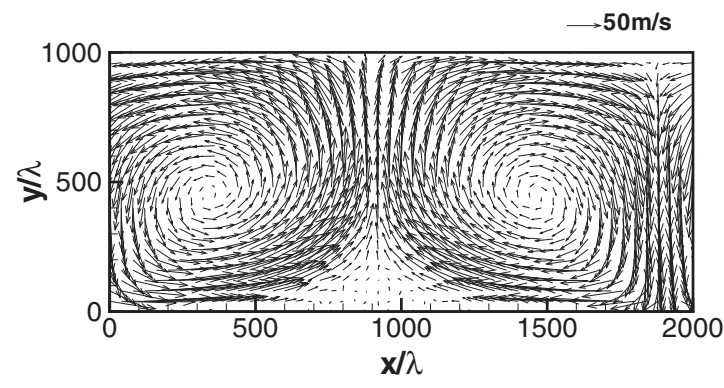

FIG. 7. The velocity vector field for $\mathrm{Ra}=1.3 \times 10^{6}$.

above-mentioned results, we can conclude that the critical vortex scale will be somewhat different under different conditions, but generally the effective diffusion will be reduced when the convection scale is about $10 \lambda$ due to the dominant boundary effect in such small scale convection.

\section{DIFFUSIVE TRANSPORT PROPERTIES IN RAYLEIGH-BÉNARD CONVECTION}

Another classical convection flow is RB convection, where molecules are confined between two horizontal parallel walls maintained at two different temperatures, and molecules are subjected to external force. Convection vortices will form above the onset of thermal instability. As stated in previous papers, ${ }^{14,16}$ the Rayleigh number is dependent on the Knudsen number, the Froude number and the temperature ratio of the upper wall to that of the lower wall. A direct and interesting idea is to study the transport properties for different scales of vortex in RB convection. However, DSMC results ${ }^{14,25}$ and linear stability analysis ${ }^{26}$ based on compressible Navier-Stokes equations have showed that there is a critical scale of the vortex about 30 times of molecular mean free paths in RB convection, that is, the thermal convection will not occur if the characteristic length of the RB system is less than the critical scale. Therefore, the phenomenon of diffusion reduction does not exist in RB convection. Definitely, we have found the diffusion will be enhanced as long as there is convective vortex structure in RB convection. In this section, our studies focus on the diffusive transport properties in the continuum regime. Our simulation is performed under the condition of $\mathrm{Kn}=0.001$, that is, the characteristic scale of convection vortex is 1000 mean free paths. Under such condition, it is obvious that the effect of solid wall on diffusion becomes negligible based on results in the previous section. In the simulation, we also fix the temperature ratio at 0.1 , and the variation of Rayleigh number is realized by adjusting the Froude number. Diffusive reflections are assumed at the upper and lower walls, while periodic boundary conditions are adopted at both sides in the horizontal directions. The two-dimensional computational domain is divided into $200 \times 100$ sampling cells within which the filed quantities are obtained by sampling the molecular information, and each sampling cell contains enough subcells within which collision pairs are selected. The calculation time step $\Delta t$ equals to $0.1 \tau$, where $\tau$ is the mean collision time of gaseous molecules. After a long enough transient period, the flow field arrives at a conductive state or convective state depend-
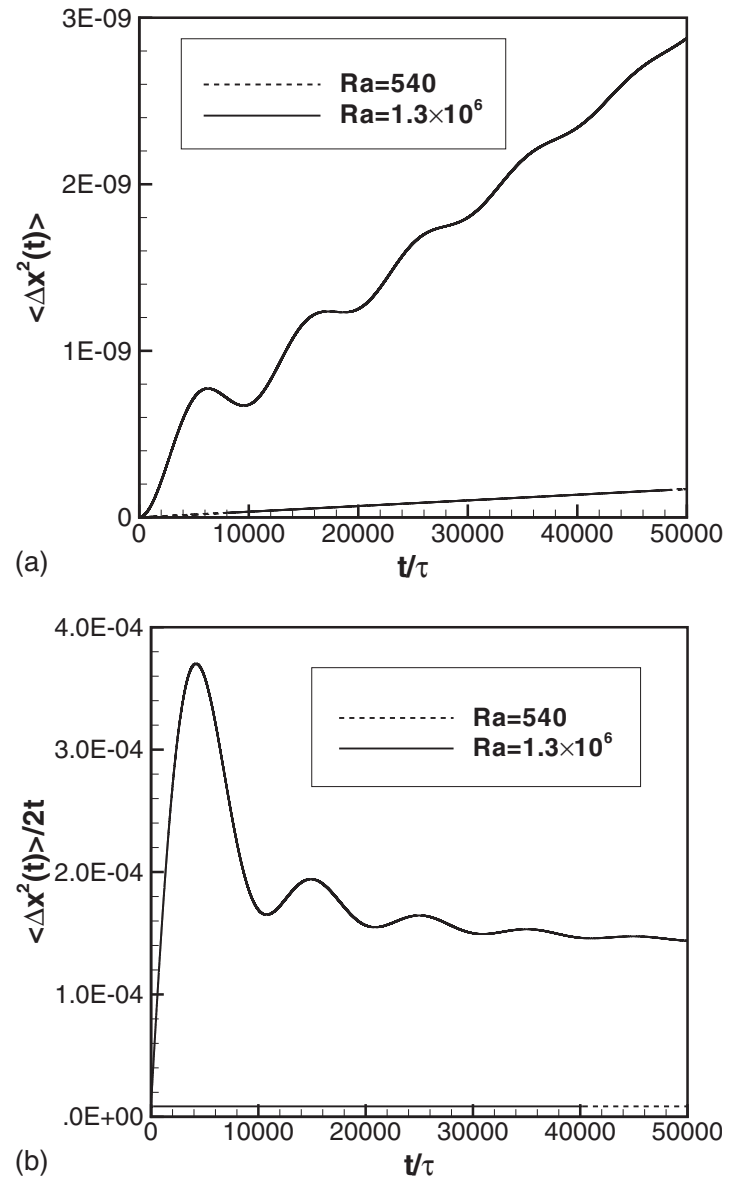

FIG. 8. The mean square displacements (a) and the ratio $\left\langle\Delta x^{2}(t)\right\rangle / 2 t$ (b) for $\mathrm{Ra}=540$ and $\mathrm{Ra}=1.3 \times 10^{6}$.

ing on the Rayleigh number, and then the molecular mean square displacement in the horizontal direction can be obtained by averaging the displacements of all molecules.

The simulation results obtained by DSMC have showed that the critical Rayleigh number is about $1773 .{ }^{14,16}$ Here we first consider two cases: one is in a thermal conductive state for $\mathrm{Ra}=540$; the other is in a steady thermal convective state for $\mathrm{Ra}=1.3 \times 10^{6}$, where the velocity vector distributions are shown in Fig. 7. The corresponding molecular mean square displacements in the horizontal direction are shown in Fig. 8 (a). For $\mathrm{Ra}=540$, the increase of mean square displacement quickly becomes linear because there is only molecular diffusion. For $\mathrm{Ra}=1.3 \times 10^{6}$, the mean square displacement is much larger than that for a conduction state due to the convection effect. In order to obtain the effective diffusion coefficient, the ratio $\left\langle\Delta x^{2}(t)\right\rangle / 2 t$ is calculated and shown in Fig. 8 (b). For $\mathrm{Ra}=1.3 \times 10^{6}$, the ratio increases rapidly at first because molecules are accelerated by the flow field, and then reaches a maximum at about $t / \tau=4000$, afterwards the ratio oscillates and gradually attenuates. This is the intermediate time scale when some molecules come back near their original positions in the process of advection along convective streamlines. After the molecules are sufficiently diffused, the ratio arrives at a constant value, which is the effective diffusion coefficient $D^{*}$ for the convective state. For $\mathrm{Ra}=540$, the effective diffusion coefficient almost equals to the molecular 


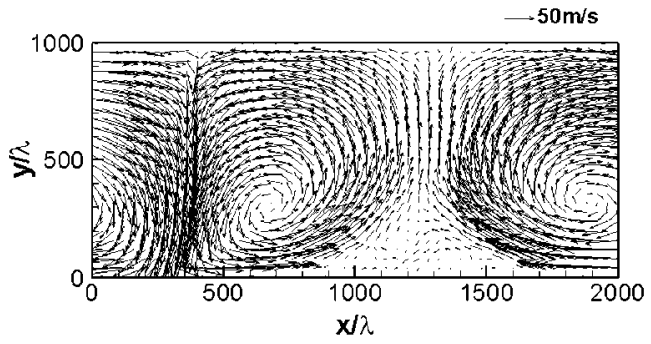

(a) $t / \tau=10000$

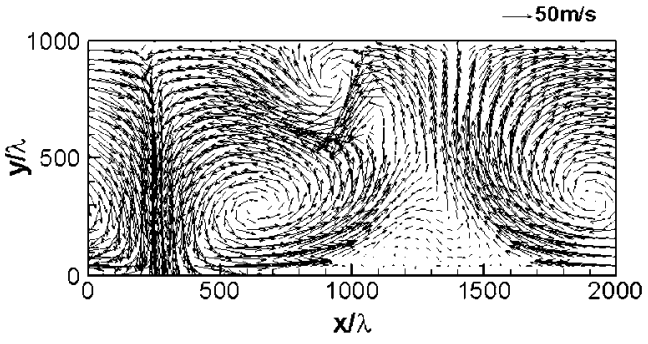

(c) $t / \tau=16000$

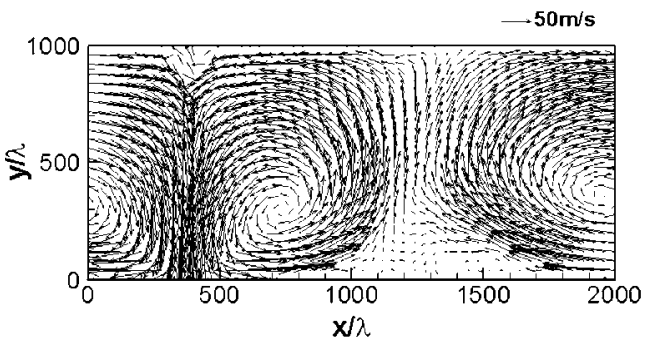

(e) $t / \tau=22000$

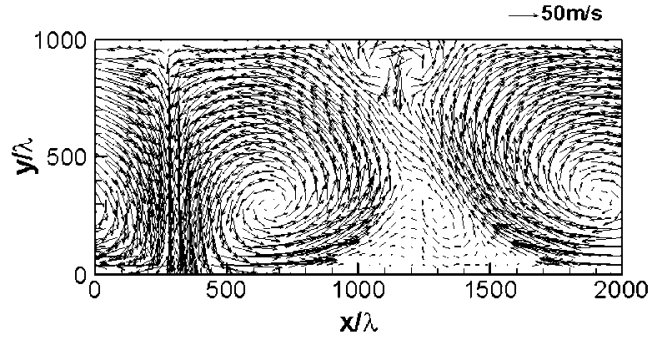

(b) $t / \tau=14000$

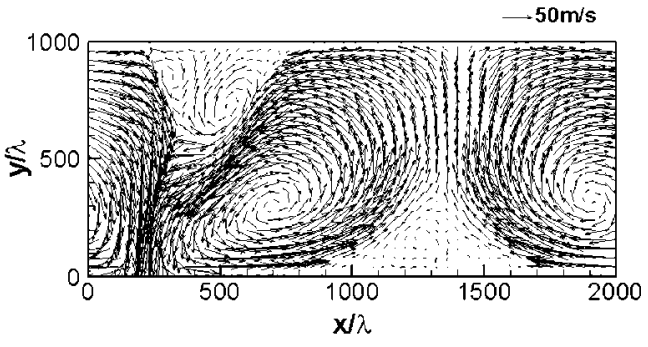

(d) $t / \tau=18000$

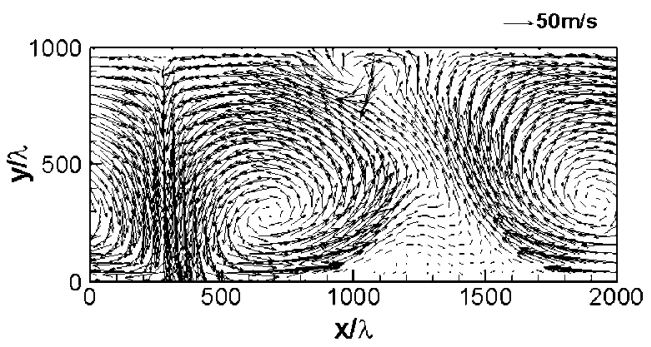

(f) $t / \tau=26000$

FIG. 9. A series of velocity vector field snapshots representing oscillation behavior for $\mathrm{Ra}=4.3 \times 10^{6}$.

diffusion corresponding to the average temperature in the field. Consequently, the diffusion enhancement due to the convection can be determined by using Eq. (3). For $\mathrm{Ra}=1.3 \times 10^{6}, \delta_{1}=15.45$; for $\mathrm{Ra}=540, \delta_{1}=0.0$.

In the continuum regime, McCarty and Horsthemke ${ }^{27}$ showed that $\delta_{1}$ was proportional to $\mathrm{Pe}^{1 / 2}$ by using homogenization method for large Péclet number (Pe) in twodimensional periodic convection flows, where temperature is uniform everywhere in the field. The Pe number describes the relative strength of the convection to the molecular diffusion, namely,

$$
\mathrm{Pe}=U d / D_{m},
$$

where $U$ and $d$ are the characteristic velocity and length of the convective flow, respectively. We use four simulation results obtained by DSMC in steady convection to make a power-law fit. The characteristic velocity in Pe is determined by the average velocity in the horizontal direction of the flow field. The result of the estimation reads

$$
\delta_{1}=1.08 \mathrm{Pe}^{0.514},
$$

which is in relatively good agreement with the theoretical prediction. ${ }^{27}$ Based on the comparison between our result and the theoretical prediction, we can conclude that in large- scale Rayleigh-Bénard convection, the effect of convection on diffusion is dominant.

In the steady convection regime, the diffusion enhancement arises mainly from the advection of molecules along the streamlines within convection rolls, while the diffusion between adjacent rolls is dependent only on the molecular diffusion, thereby the diffusion enhancement is limited. Therefore, there is a great gap between the limited diffusion enhancement in the steady convection regime and the turbulent diffusion. More powerful diffusion needs new mechanism to enhance advection, and the oscillation convection for large Rayleigh number may have the potential. A series of snapshots presented in Fig. 9 show some typical evolutions of the velocity vector field for $\mathrm{Ra}=4.3 \times 10^{6}$ after the convection has formed. Different from the steady convection for $\mathrm{Ra}=1.3 \times 10^{6}$, the established flow oscillates with time quasiperiodically. Correspondingly, the molecular mean square displacement and effective diffusion coefficient shown in Fig. 10 for $\mathrm{Ra}=4.3 \times 10^{6}$ are larger than those found in the steady convection. This is because molecules in oscillation convection can easily advect from one roll to another when the roll boundaries oscillate. In this way, diffusion between rolls depends not only on the molecular thermal motion but 


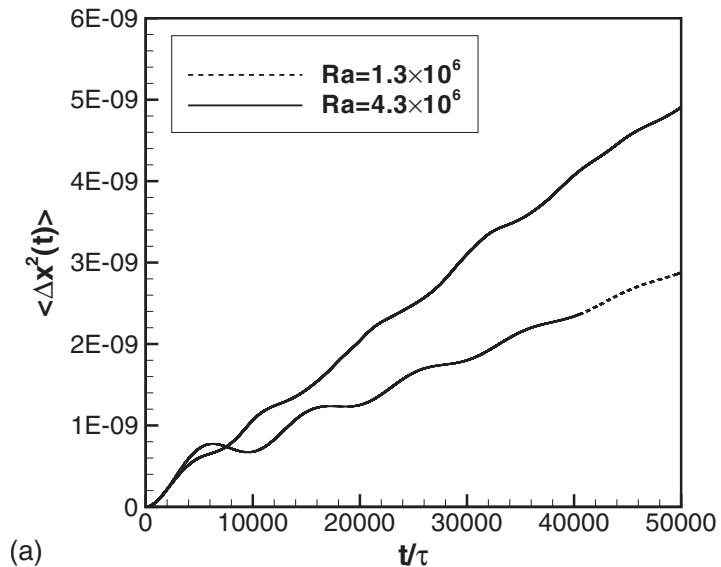

(a)

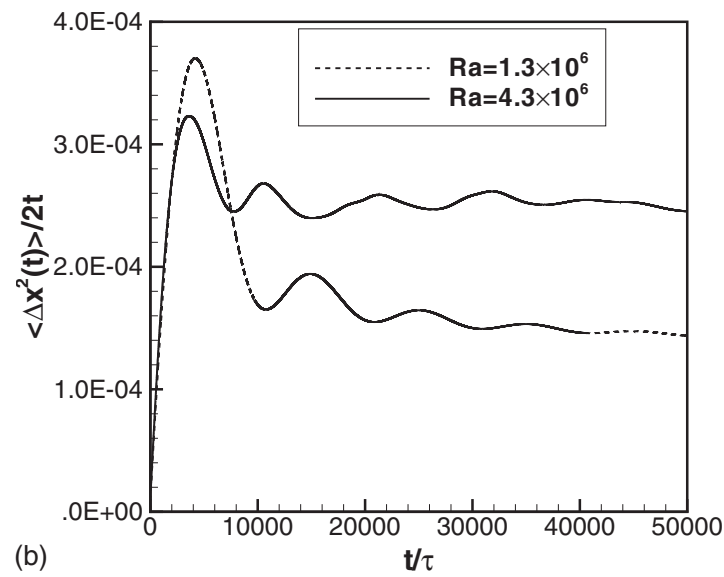

FIG. 10. The mean square displacements (a) and the ratio $\left\langle\Delta x^{2}(t)\right\rangle / 2 t$ (b) for $\mathrm{Ra}=4.3 \times 10^{6}$ and $\mathrm{Ra}=1.3 \times 10^{6}$

also on the advection transport, and thus the transport is greatly enhanced.

For convenient comparison, the diffusion enhancements of different convection regimes against Pe are shown in Fig. 11 , from which it is obvious that diffusions in the oscillation convection are more strongly enhanced than that in the steady convection. Meanwhile, from the two cases of oscillation convection we can see that the increasing rate of diffusion enhancement with $\mathrm{Pe}$ is also larger than that in the time-independent convection. It should be noted that in our

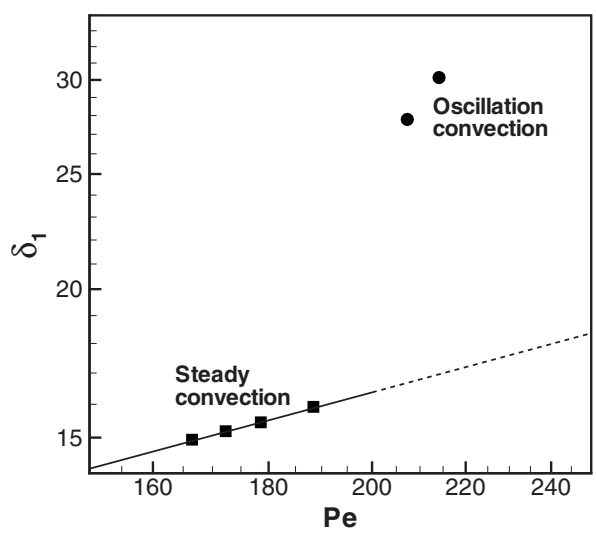

FIG. 11. Comparison of diffusion enhancement for different convection regimes. simulation we have focused on the steady convection and the oscillation convection only. Therefore, anomalous diffusion characteristic that may occur in chaotic convection ${ }^{28}$ has not been observed.

\section{CONCLUSIONS}

In this paper, diffusive transport properties in thermal creep convection and $\mathrm{RB}$ convection are investigated by using DSMC method. Our simulation results in thermal creep convection in noncontinuum regime show that the effective diffusion is enhanced only when the vortex scale exceeds a certain critical value, while the diffusion is reduced when the vortex scale is less than the critical value. The reason of diffusion reduction is that the reduction effect due to solid wall is dominant and the enhancement effect arising from convection is negligible when the convection vortex scale is less than dozens of molecular mean free path. The knowledge of diffusion reduction in noncontinuum regime shown in this paper is an instructive supplement to the traditional viewpoint that convection flows would enhance diffusion. It is especially important for the fluid flow in MEMS systems. As a preliminary study, we have focused only on diffusive transport properties, and further studies will be performed on the momentum and energy transport by calculating the effective viscosity and thermal conduction coefficients.

On the other hand, if convection flows are in the continuum regime, the effect of convection on diffusion is dominant while the effect of solid wall on diffusion is negligible. The present results in $\mathrm{RB}$ convection show that in the steady convection, the diffusion enhancement with respect to molecular self-diffusion is proportional to $\mathrm{Pe}^{1 / 2}$, which is in agreement with previous theoretical results and experimental data. In oscillation convection, diffusion is more strongly enhanced than in steady convection because molecules can easily advect from one roll to its neighbor by crossing the oscillating roll boundaries. A natural extension of this work could be a study of enhanced diffusion in fully developed turbulent flows, where the enhanced diffusion due to fluid flow is often described phenomenologically as "eddy" diffusion. The present work proves that DSMC is a promising method to directly obtain the effective diffusion coefficient based on the Einstein diffusion equation. The only limitation to the application of DSMC is too much time required by the computations. As the computational power further develops, the DSMC method may become an important tool to obtain quantitative transport properties in turbulence.

\section{ACKNOWLEDGMENTS}

This work was supported by the National Natural Science Foundation of China (Grant Nos. 10921062 and 11002147).

${ }^{1}$ G. Taylor, "Dispersion of soluble matter in solvent flowing slowly through a tube," Proc. R. Soc. London, Ser. A 219, 186 (1953).

${ }^{2}$ G. E. Karniadakis and A. Beskok, Micro Flows: Fundamentals and Simulation (Springer-Verlag, Berlin, 2002).

${ }^{3}$ K. Shida and W. G. Hoover, "Maxwell's thermal creep in two space dimensions,” J. Phys. Soc. Jpn. 67, 2277 (1998). 
${ }^{4}$ C. K. Chen and H. C. Weng, "Developing natural convection with thermal creep in a vertical microchannel," J. Phys. D 39, 3107 (2006)

${ }^{5}$ E. H. Kennard, Kinetic Theory of Gases (McGraw-Hill, New York, 1938).

${ }^{6}$ G. A. Bird, Molecular Gas Dynamics and Direct Simulation of Gas Flows (Clarendon, Oxford, 1994).

${ }^{7}$ E. L. Koschmieder, Benard Cells and Taylor Vortices (Cambridge University Press, London, 1993).

${ }^{8}$ M. Mareschal, M. M. Mansour, A. Puhl, and E. Kestemont, "Molecular dynamics versus hydrodynamics in a two-dimensional Rayleigh-Bénard system," Phys. Rev. Lett. 61, 2550 (1988).

${ }^{9}$ A. Puhl, M. M. Mansour, and M. Mareschal, "Quantitative comparison of molecular dynamics with hydrodynamics in Rayleigh-Bénard convection," Phys. Rev. A 40, 1999 (1989).

${ }^{10}$ A. García and C. Penland, "Fluctuating hydrodynamics and principal oscillation pattern analysis," J. Stat. Phys. 64, 1121 (1991)

${ }^{11}$ T. Watanabe, H. Kaburaki, M. Machida, and M. Yokokawa, "Growth of long-range correlations in a transition between heat conduction and convection," Phys. Rev. E 52, 1601 (1995).

${ }^{12} \mathrm{E}$. Golshtein and T. Elperin, "Convective instabilities in rarefied gases by direct simulation Monte Carlo method," J. Thermophys. Heat Transfer 10, 250 (1996).

${ }^{13}$ T. Watanabe and H. Kaburaki, "Particle simulation of three-dimensional convection patterns in a Rayleigh-Bénard system," Phys. Rev. E 56, 1218 (1997).

${ }^{14}$ S. Stefanov, V. Roussinov, and C. Cercignani, "Rayleigh-Bénard flow of a rarefied gas and its attractors. I. Convection regime," Phys. Fluids 14, 2255 (2002)

${ }^{15}$ S. Stefanov, V. Roussinov, and C. Cercignani, "Rayleigh-Bénard flow of a rarefied gas and its attractors. II. Chaotic and periodic convective regimes," Phys. Fluids 14, 2270 (2002).
${ }^{16}$ J. Zhang and J. Fan, "Kinetic study of the Rayleigh-Bénard flows," Chin. Sci. Bull. 54, 364 (2009)

${ }^{17}$ S. Stefanov, V. Roussinov, and C. Cercignani, "Rayleigh-Bénard flow of a rarefied gas and its attractors. III. Three-dimensional computer simulations," Phys. Fluids 19, 124101 (2007).

${ }^{18}$ S. Vannitsem and M. Mareschal, "Molecular dynamics simulations of passive transport in two-dimensional Rayleigh-Bénard convection," Phys. Rev. E 51, 5564 (1995).

${ }^{19}$ T. H. Solomon and J. P. Gollub, "Passive transport in steady RayleighBénard convection," Phys. Fluids 31, 1372 (1988).

${ }^{20}$ J. P. Boon and S. Yip, Molecular Hydrodynamics (McGraw-Hill, New York, 1980).

${ }^{21}$ J. O. Hirschfelder, C. F. Curtiss, and R. B. Bird, Molecular Theory of Gases and Liquids (Wiley, New York, 1954).

${ }^{22}$ K. Aoki, P. Degond, S. Takata, and H. Yoshida, "Diffusion models for Knudsen compressors," Phys. Fluids 19, 117103 (2007).

${ }^{23}$ T. Ohwada, Y. Sone, and K. Aoki, "Numerical analysis of the shear and thermal creep flows of a rarefied gas over a plane wall on the basis of the linearized Boltzmann equation for hard-sphere molecules," Phys. Fluids A 1, 1588 (1989)

${ }^{24}$ D. H. Papadopoulos and D. E. Rosner, "Enclosure gas flows driven by nonisothermal walls," Phys. Fluids 7, 2535 (1995).

${ }^{25}$ J. Zhang and J. Fan, "Monte Carlo simulation of thermal fluctuations below the onset of Rayleigh-Bénard convection," Phys. Rev. E 79, 056302 (2009)

${ }^{26}$ A. Manela and I. Frankel, "On the Rayleigh-Bénard problem in the continuum limit," Phys. Fluids 17, 036101 (2005).

${ }^{27}$ P. McCarty and W. Horsthemke, "Effective diffusion coefficient for steady two-dimensional convective flow," Phys. Rev. A 37, 2112 (1988).

${ }^{28}$ T. H. Solomon and J. P. Gollub, "Chaotic particle transport in timedependent Rayleigh-Bénard convection," Phys. Rev. A 38, 6280 (1988). 